\title{
BMJ Open Intimate partner violence and breastfeeding: a systematic review
}

\author{
Anne Katrine Normann (D , ${ }^{1}$ Aleksandra Bakiewicz, ${ }^{1}$ Frederikke Kjerulff Madsen, ${ }^{1}$ \\ Khalid Saeed Khan, ${ }^{2}$ Vibeke Rasch, ${ }^{3,4}$ Ditte Søndergaard Linde (D) ${ }^{3,4}$
}

To cite: Normann AK, Bakiewicz A, Kjerulff Madsen F, et al. Intimate partner violence and breastfeeding: a systematic review. BMJ Open 2020;10:e034153. doi:10.1136/ bmjopen-2019-034153

- Prepublication history and additional materials for this paper is available online. To view these files, please visit the journal online (http://dx.doi. org/10.1136/bmjopen-2019034153).

Received 07 September 2019 Revised 11 August 2020 Accepted 21 September 2020

Check for updates

(C) Author(s) (or their employer(s)) 2020. Re-use permitted under CC BY-NC. No commercial re-use. See rights and permissions. Published by BMJ.

${ }^{1}$ Department of Clinical Research, University of Southern Denmark, Odense, Denmark ${ }^{2}$ Department of Preventive Medicine and Public Health, Faculty of Medicine, University of Granada, Granada, Spain

${ }^{3}$ Obstetrics and Gynaecology, Odense Universitetshospital, Odense, Denmark

${ }^{4}$ Institute of Clinical Research, Syddansk Universitet, Odense Denmark

Correspondence to Anne Katrine Normann; annekatrinenn@gmail.com

\section{ABSTRACT}

Objective The association between intimate partner violence (IPV) and breastfeeding is unclear. We conducted a systematic review to summarise the evidence of breastfeeding outcomes following exposure to IPV.

Design Systematic review.

Methods We searched for published studies without study design or language restrictions (up to July 2019) in the following databases: PubMed, Embase, SCOPUS and The Global Health Library. Studies assessing various breastfeeding outcomes (initiation, duration and exclusive breastfeeding) in women exposed to IPV in any form (physical, psychological or sexual) and at any stage (1 year pre-pregnancy, during or post-pregnancy) were included. Two authors independently selected the studies and conducted the quality appraisal by use of the NewcastleOttawa Scale. Results were summarised taking precision and quality into account.

Results A total of 16 studies (participants $n=414393$ ) were included and they adjusted for a total of 48 different confounders. The majority of studies were crosssectional $(n=11)$ and most studies were judged to be fair/low quality. Four out of seven studies found that IPV exposure shortened breastfeeding duration (adjusted ORs/a0Rs=0.22 (95\% Cl: 0.05-0.85), 1.18 (95\% Cl: 1.01-1.37), 5.92 (95\% Cl: 1.72-27.98), 1.28 (95\% Cl: 1.18-1.39)). Further, $5 / 10$ studies found that IPV led to early termination of exclusive breastfeeding (aORs $=1.53$ (95\% Cl: 1.01-23.1), 0.83 (95\% Cl: 0.71-0.96), 1.35 (95\% Cl: $1.07-1.71), 0.17$ (95\% Cl: 0.07-0.4), 1839 (95\% Cl: 1.61-2911)) and 2/6 studies found that IPV significantly reduced breastfeeding initiation $(\mathrm{aORs}=2.00(95 \% \mathrm{Cl}$ : 1.2-3.3), 0.81 (95\% Cl: 0.7-0.93)).

Conclusion IPV exposure appears to associate negatively with some breastfeeding outcomes. Individual patient data meta-analysis is required to quantify the magnitude of the association for specific IPV-outcome combinations. More high-quality studies and definition of core confounders are warranted.

PROSPERO registration number CRD42019129353.

\section{INTRODUCTION}

Intimate partner violence (IPV) is defined as any behaviour by a current or former intimate partner that causes physical, psychological or sexual harm to those within an intimate relationship. ${ }^{12}$ Most often, IPV is perpetrated by men against women, ${ }^{1-3}$ and the WHO estimates that one in three women
Strengths and limitations of this study

This systematic review provides the latest evidence of the association between intimate partner violence and breastfeeding.

- Our review excluded studies with women who had a lifetime history of violence and childhood abuse.

- We conducted an appropriate quality assessment of studies by use of the Newcastle-0ttawa Scale.

- The heterogeneous field of confounders in the included studies was grouped by making key domains.

- It was not possible to make a causal association nor conduct a meta-analysis.

will be exposed to either physical/or sexual violence during their lifetime. ${ }^{2}$ IPV can have both immediate and long-term mental and physical health consequences for the victims, including depression and physical impairment. ${ }^{3-5}$ Further, it has been found that IPV is related to a number of reproductive health outcomes, including preterm birth, low birth weight, insufficient weight gain, miscarriage, induced abortion and difficulties or lack of attachment to the baby. ${ }^{3-6}$ It has been speculated that IPV may also influence the establishment of breastfeeding practices, however this association is complex. The WHO recommends initiating breastfeeding within 1 hour of birth, exclusive breastfeeding for 6 months and that mothers should continue breastfeeding for up to 2 years or beyond together with complementary feeding. ${ }^{7}$ IPV may affect breastfeeding directly, for example, through sore nipples and difficulty in relaxing enough for adequate let-down, but also indirectly, for example, through lack of support or depression, self-doubt, body negativity and anxiety. ${ }^{8}$ Furthermore, qualitative studies have found that women who have experienced violence in their childhood may have trouble continuing exclusive breastfeeding due to difficulties in separating the sexual role from the maternal role of breasts or due to lack of situational control. ${ }^{10}{ }^{11}$ The existing literature is characterised by various 
outcome measures for both IPV and breastfeeding, and further there is no agreement of core factors that may confound the association between IPV and breastfeeding, hence, there is variation in the statistical models used for analysing the relationship. This may be the reasons why studies on the relationship of IPV and breastfeeding practice have had inconsistent results. A study of IPV and breastfeeding practices across Africa found that IPV was associated with lower adjusted odds for breastfeeding initiation and exclusive breastfeeding in some African countries and higher adjusted odds in other countries, ${ }^{12}$ while a recent systematic review concluded that the majority of studies ( $n=12$, participant=133861) found a negative association between breastfeeding initiation and exclusive breastfeeding for the first 6 months. ${ }^{13}$ Yet, the review did not involve an appropriate quality assessment and had no detailed discussion of confounders. Further, new literature has been published. Therefore, we conducted a robust systematic review thoroughly investigating the association of exposure to IPV pre-pregnancy, during and post-pregnancy with breastfeeding outcomes and synthesised the evidence taking confounders, precision and quality into considerations.

\section{METHODS}

This systematic review was conducted according to the Preferred Reporting Items for Systematic Reviews and Meta-Analyses $^{14}$ and Meta-analyses Of Observational Studies in Epidemiology ${ }^{15}$ guidelines.

\section{Eligibility criteria and search methods identification of studies}

We searched PubMed, Embase, SCOPUS and the Global Health Library from 8 to 12 March 2019 with no time or language restrictions. An updated search was conducted on the 18th of July 2019. Search terms included "intimate partner violence" OR "spouse abuse" OR "domestic violence" OR "physical abuse" OR "sex offenses" OR "battered women" AND "breastfeeding" OR "breastmilk expression" OR "feeding behavior" OR "milk, human" OR lactation OR "milk ejection" (full search in online supplemental appendix S1).

A PICO (population, intervention, control and outcomes), model was made to develop the search strategy and selection of the literature. ${ }^{16}$ We included studies with women exposed to violence 1 year prior to pregnancy, during pregnancy and in the postpartum period which met the following criteria: (a) men as perpetrators of violence against women, (b) women in an intimate relationship over 1 month during previous pregnancies, current pregnancy and post partum, (c) women who breastfed from the first hour and until 6 months after giving birth, (d) women exposed to IPV but also perpetrators of violence against men, (e) women exposed to other forms of violence (eg, gang violence, bullying). We excluded (a) women in intimate relationships of less than 1 month of duration (during previous pregnancies, current pregnancy or post partum), (b) women who gave birth to twins or triplets, (c) women with absolute counterindication for breastfeeding, (d) women who were not able to breastfeed (eg, due to mastectomy), (e) women with eating disorders or chronic illness (eg, HIV), (f) women with substance abuse (eg, alcohol, drugs), (g) studies with only sexual minorities (eg, bisexual, homosexuals).

IPV was defined as the following: physical violence (ie, slapping, hitting, kicking, beating), sexual violence (including forced sexual intercourse or other forms of sexual coercion), psychological violence (humiliation, insults, intimidation, threats of harm), economic violence (ie, restricting access to financial resources, education, employment and medical care) and controlling behaviours (ie, isolating a person from friends and family, controlling their movements, restricting access to education and employment).

Outcome was breastfeeding practices in according with the WHO's recommendations defined as: (a) intention to breastfeed (when the woman showed interest in offering breast milk), (b) start of breastfeeding/duration (when the woman offered the child breast milk in the postpartum period), (c) exclusive breastfeeding of children from first day of life and up to 6 months (exclusive breastfeeding defined as the infant only receiving breast milk without any additional food or drink, not even water), (d) duration of exclusive breastfeeding. Eligible studies for inclusion were original publications of observational studies.

After removing duplicates, two authors independently screened titles, abstract and full text (AKN and $\mathrm{AB})$ using Covidence (www.covidence.org). ${ }^{17}$ Disagreements were solved through discussion. One author (AKN) extracted data from included studies into a standardised Excel template. Extracted data included: Title, first author, publication year, country, study characteristics, study objective, participant characteristics, sample size, inclusion/exclusion criteria, type of exposure, measurement tool of exposure, primary outcomes and confounders that were adjusted for in the statistical analysis. Outcome data were verified by a second author (FKM).

\section{Study quality assessment and data synthesis}

The Newcastle-Ottawa Scale (NOS) was used to assess the quality of cohort studies and a modified version of the scale was used for cross-sectional studies. The scale addresses the following domains: Selection process, comparability, exposure and outcome of interest. A maximum of 9 stars can be given if all domains are well described in a given study. Cohort studies are regarded to be of 'good quality' if rewarded 3 or 4 stars in the selection domain, 1 or 2 stars in the comparability domain, and 2 or 3 stars in the outcome/exposure domain. Studies are regarded as being of 'fair quality' if rewarded 2 stars in the selection domain, 1 or 2 stars in the comparability domain, and 2 or 3 stars in the outcome/exposure domain. Finally, studies are judged as being of 'poor quality' if rewarded 0 or 1 star in the selection domain or 0 stars in the comparability domain or 0 or 1 stars in the outcome/exposure 
domain. For the cross-sectional version of the scale, the domain that assessed confounders was modified and no stars were given if studies did not justify their choice of confounders in their statistical analysis. A total of 10 stars can be given to the cross-sectional studies, and they are regarded as 'very good' if rewarded 9-10 points, 'good' if rewarded 7-8 points, 'satisfactory' if rewarded 5-6 points and 'unsatisfactory' if rewarded $0-4$ points. Further, we modified both scales and added a point system for confounders, so that each study was given 1 point or star for each confounder they adjusted for (see online supplemental table S2.1) Two authors (AKN and FKM) conducted the quality assessment independently and compared results. Disagreements were solved through discussion. AKN and DSL conducted the quality assessment of Madsen et al, as FKM was coauthor of this study and therefore considered ineligible.

Two authors (AKN and DSL) made key domains for confounding factors and grouped them (see online supplemental table S2.2). Results for the various IPV outcome combinations were summarised and physical violence was presented in a forest plot. Studies that investigated physical violence were presented to emphasise the most reported form of IPV. ${ }^{3}$ Inferences were generated taking study precision and quality into account as metaanalysis was not possible. The substantial heterogeneity of exposure, outcome, study quality and statistical models in the adjusted ORs (aORs) reported in individual studies was the reason we settled for a qualitative synthesis in the form of vote-counting, which we conducted within broad exposure-outcome subgroups stratified by study quality and precision to minimise bias. To determine whether a study showed a negative association or no difference, we relied on numerical data in vote-counting to avoid subjectivity. This approach is in line with what is considered suitable given study variability in previous review publications. 1819

\section{Patient and public involvement \\ No patients involved.}

\section{RESULTS}

The database searches resulted in 2062 records and 1634 records were eligible for title and abstract screening after removal of duplicates (figure 1). A total of 16 studies met the inclusion criteria of which 11 were cross-sectional ${ }^{20-31}$ and 5 were cohort studies. ${ }^{32-35}$ The studies were published between 2006 and 2019. Four studies were conducted in the USA, ${ }^{20} 222531$ four in India, ${ }^{21} 232629$ two in Brazil, ${ }^{28} 34$ one in Tanzania, ${ }^{32}$ one in Spain, ${ }^{33}$ one in Sweden, ${ }^{24}$ one in Norway, ${ }^{35}$ one in Australia ${ }^{27}$ and one in Hong Kong. ${ }^{30}$ Population age ranged from 14 to 49 years and was reported as means $(n=3)$ or in intervals $(n=13)$. The size of the study population varied from 69 to 195264 participants with a mean sample size of 25899 (table 1). Exposure (IPV) was measured through questionnaires 22232526293132 or through various validated tools: The Conflict-Tactic

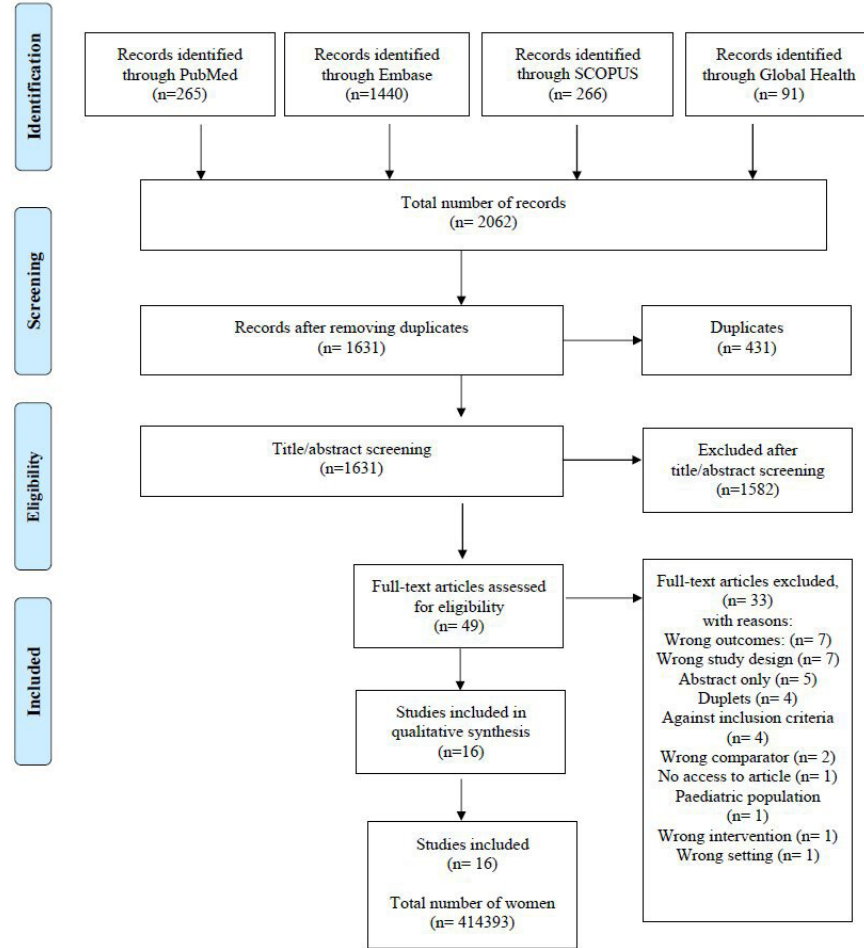

Figure 1 Flow chart of study selection in the review of intimate partner violence and breastfeeding outcomes.

Scale,,$^{20} 283034$ the Abuse Assessment Screen, ${ }^{30}$ the Index of Spouse Abuse, ${ }^{33}$ the Composite Abuse Scale ${ }^{27}$ and the Norvold Abuse Questionnaire ${ }^{24}$ (table 1).

In regards to exposure, two of the studies only focused on physical violence ${ }^{22} 28$ and one study only focused on psychological violence. ${ }^{21}$ However, the majority of studies measured IPV as 'any IPV' and did not separate violence into groups, ${ }^{2022} 242729-3134$ and five studies measured both physical, or/and psychological or/and sexual violence, respectively, and combined to compare the differences in exposure of a certain type of IPV. ${ }^{2326} 323335$ The outcome breastfeeding was measured as early cessation/shortened duration of breastfeeding, initiation of breastfeeding or exclusive breastfeeding. The definition of 'shortened duration of breastfeeding' differed as each study set their own time limit (see online supplemental table S2.3). Some studies investigated more than one outcome and therefore, one study could be presented in more than one outcome table.

Overall, the included studies adjusted for 48 different confounders within the following domains: maternal sociodemographic, relationship characteristics, maternal lifestyle and health, economy, pregnancy and postpartumrelated problems, child characteristics, support during pregnancy and post partum, violence or stressful life events, pregnancy intention, caste and religion. The most common confounding factors were maternal lifestyle and health, maternal sociodemographics and relationship characteristics. The majority of studies did not justify their choice of confounders. ${ }^{21-25} 2729-3134$ Sorbo et al and Madsen et al used the directed acyclic graph to justify the 


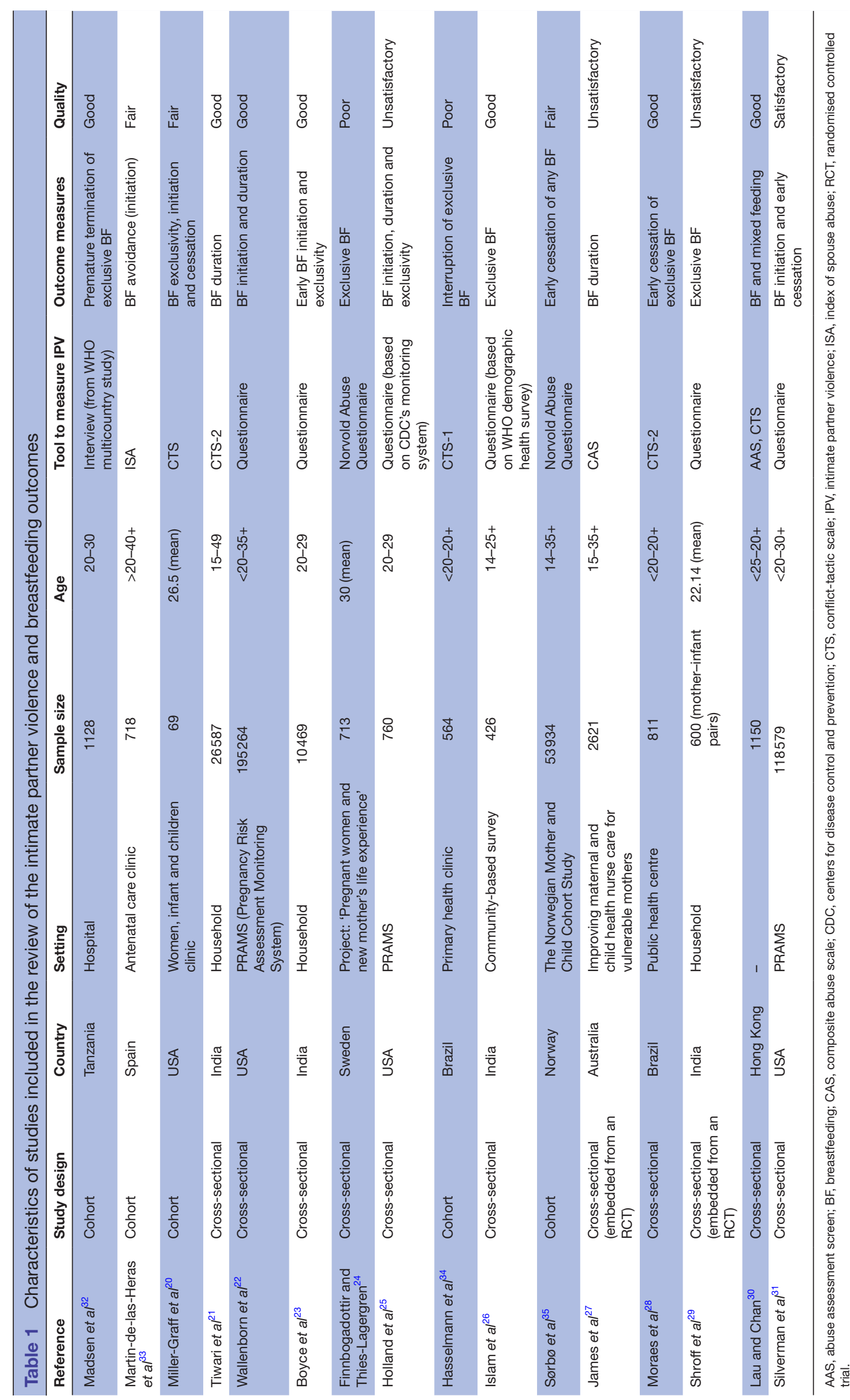


confounders adjusted for in their analysis, and Sorbo et al also made a sensitivity analysis to determine whether or not the association between IPV and breastfeeding practices was mediated primarily through postpartum depression. They found that depression could not explain early cessation of breastfeeding. ${ }^{35}$

\section{Study quality assessment}

Of the five cohort studies, one study was judged as 'good quality', ${ }^{32}$ three studies was judged as 'fair quality'20 3335 and one study was judged as 'poor quality'. ${ }^{34}$ Of the 11 cross-sectional studies, six were judged as having 'good' quality, ${ }^{21-23262830}$ one was judged as 'satisfactory',31 and three studies were judged as 'unsatisfactory'. 252729 One cross-sectional study was not assessed using NOS for crosssectional studies, since the study was embedded from a cohort, ${ }^{24}$ hence, NOS for cohort studies was used to assess the quality and it was judged as of "poor quality ${ }^{24}$ (figure 2).

\section{Initiation of breastfeeding}

Six studies investigated the association between exposure to IPV and initiation of breastfeeding. ${ }^{20} 2223253133$ Two studies found a statistical significant association between initiation of breastfeeding and exposure to either physical or sexual violence ${ }^{23}\left(\mathrm{aOR}_{\text {physical }}=0.81 ; 95 \%\right.$ CI: $0.71-$ $0.93 . \mathrm{aOR}_{\text {sexual }}=0.52 ; 95 \%$ CI: $\left.0.36-0.76\right)$ or psychological violence $^{33}(\mathrm{aOR}=2.00 ; 95 \% \mathrm{CI}: 1.2-3.3)$. Four studies found no association when exposed to multiple types of violence 20232531 (see online supplemental table S2.4).

\section{Shortened duration of breastfeeding}

Seven studies reported outcomes based on early cessation or shortened duration of breastfeeding when exposed to violence, ${ }^{20-22} 25273135$ and four studies found a significant association (aORs $=0.22$ (95\% CI: 0.05-0.85), 1.18 (95\% CI: 1.01-1.37), 5.92 (95\% CI: 1.72-27.98), 1.28 (95\% CI: 1.18-1.39)) between exposure to IPV and early cessation/shortened duration of breastfeeding ${ }^{20235}$ (see online supplemental table S2.3). Miller-Graff $e t a l^{20}$ found that IPV was associated with decreased OR of continuation of breastfeeding (OR=0.22; 95\% CI: 0.5-0.85), hence, IPV was associated with an increased risk of shortened duration of breastfeeding. Further, one study found a statistical significant association between reduced duration/early cessation and IPV (OR=1.41 95\% CI: 1.15-1.74). However, the association became insignificant when adjusting for confounders $(\mathrm{aOR}=0.94 ; 95 \%$ CI: $0.76-1.7) .{ }^{31}$ Three of the studies found no association between violence and breastfeeding duration or early cessation. ${ }^{21} 2527$ Three studies did not distinguish between period of exposure, ${ }^{27} 2934$ whereas the remaining papers categorised time of exposure. One study ${ }^{27}$ found no association between IPV and breastfeeding practices and concluded that IPV itself did not influence breastfeeding outcomes as much as maternal age, education and birth method (see online supplemental table S2.3).
Cohort studies

Representativeness of the exposed cohort

Selection of the non-exposed cohort

Ascertainment of exposure

Outcome of interest absent at start of study

Comparability of cohorts (design or analysis)

Confounding domains adjusted for

Assessment of outcome

Adequate length of follow-up

Adequacy of follow up of cohorts

Overall good quality

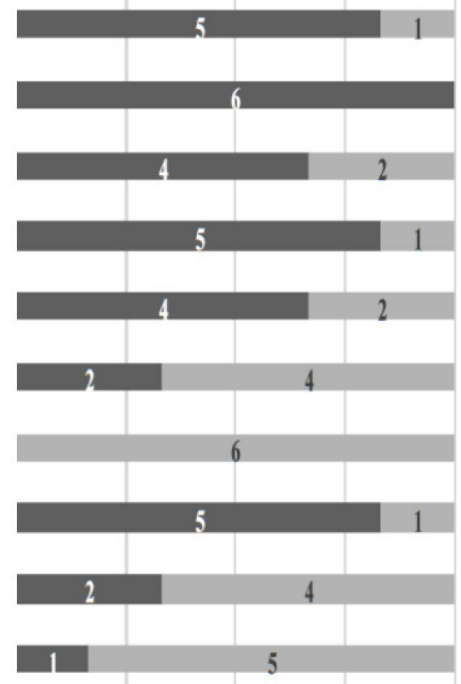

Cross -sectional studies

Representativeness of the sample

Sample size

Non-respondents

Ascertainment of the exposure (risk factor)

Comparabillity (confouding factors)

Confounding domains ajusted for

Assessment of outcome

Statistical test

0verall good quality

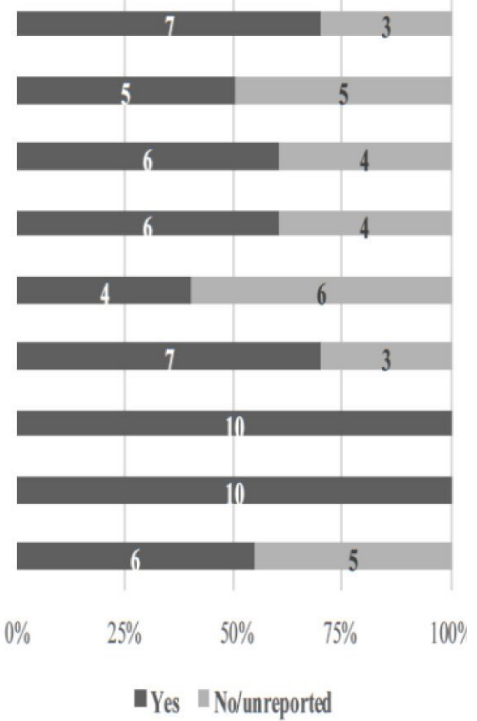

Figure 2 Study quality of cohort and cross-sectional studies in the review of intimate partner violence and breastfeeding outcomes. In the figure illustration of the Newcastle-Ottawa Scale (NOS), the studies which reached a maximum of stars in each category of the NOS was rewarded a 'yes', and further if the studies adjusted for more than four confounding domains, they were rewarded a 'yes' (see online supplemental appendix S2).

\section{Exclusive breastfeeding}

Ten studies assessed exposure to violence in relation to risk of early termination of exclusive breastfeeding and five studies found a statistical association (aORs=1.53 (95\% CI: 1.01-23.1), 0.83 (95\% CI: 0.71-0.96), 1.35 (95\% CI: $1.07-1.71), 0.17$ (95\% CI: 0.07-0.4), 1839 (95\% CI: $1.61-2911)^{2326303234}$ and five studies found no statistical association $^{20} 24252829$ (figure 3) (see online supplemental table S2.5). 


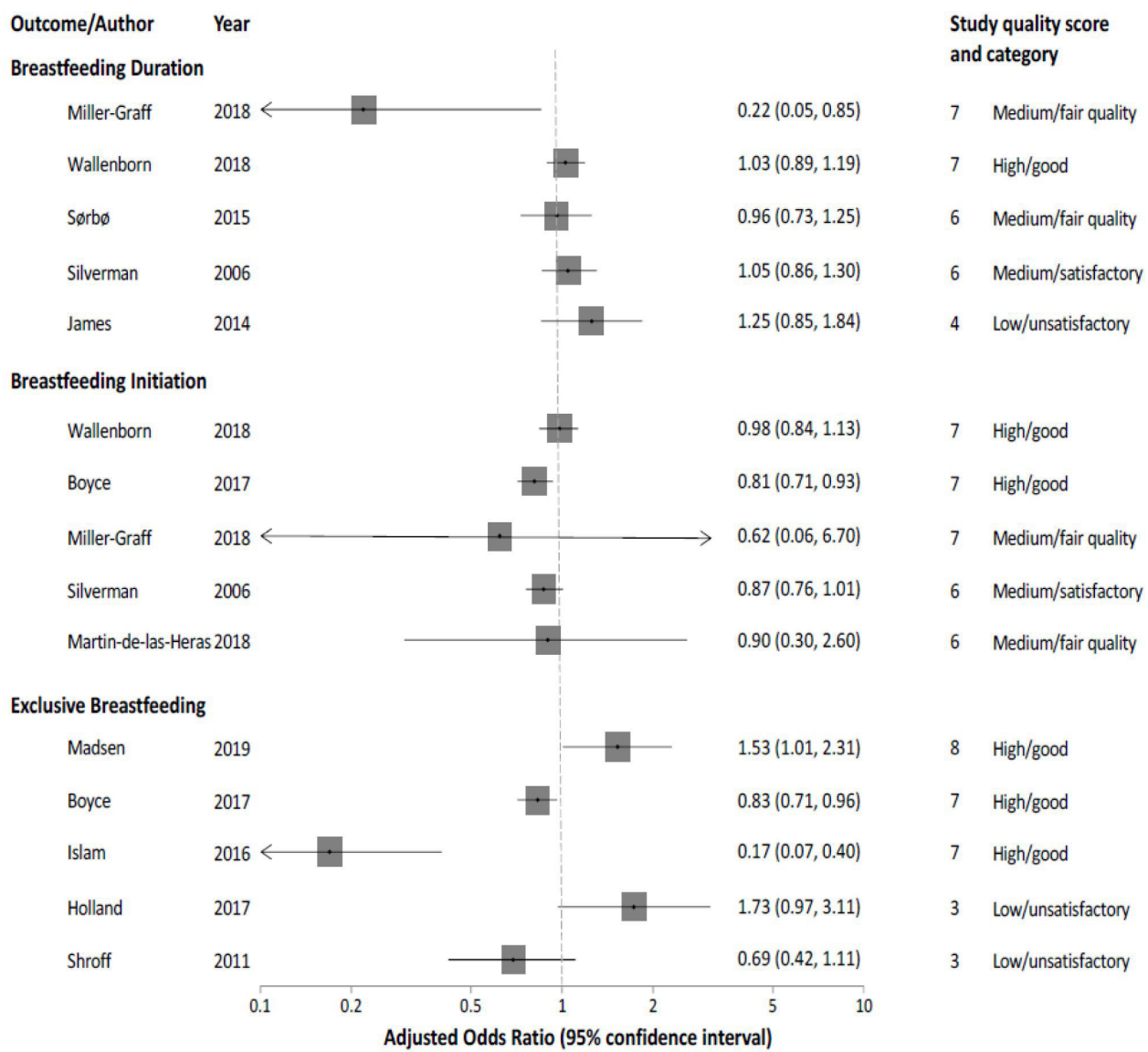

Figure 3 Results of physical violence and the association with breastfeeding duration, breastfeeding initiation and exclusive breastfeeding presented in a forest plot ordered according to descending quality.

\section{DISCUSSION}

\section{Main findings}

This systematic review summarised the most recent evidence, between exposure to IPV and breastfeeding practices. A total of 16 studies were included of which 11 were cross-sectional and 5 were cohort studies. Forty-eight different confounders were controlled for in the studies. Only one cohort was judged as being of good quality, hence, the overall quality of the studies was fair to low. The majority of studies found that exposure to IPV in any form and at any stage had a significant negative association with breastfeeding duration, early termination of exclusive breastfeeding, but it did not reduce initiation.

\section{Strengths and limitations}

The review synthesises the latest evidence of pregnancyrelated IPV and the WHO recommended breastfeeding practices and elucidates the complex association between IPV exposure, breastfeeding and confounding factors. A limitation of this review is that the majority of included studies were cross-sectional, hence, a causal association cannot be estimated, ${ }^{36}$ and we were not able to conduct a meta-analysis. Therefore, there is a need for welldesigned longitudinal studies to better estimate the association. The individual results were presented in a forest plot, without meta-analysis to illustrate the heterogeneity across studies. The forest plot was ordered in the vertical axis by the risk of bias in a manner that places higherquality study findings above those with lower quality. This approach is in line with the recommendation to exploit the plot's vertical dimension should be used to illustrate differences in important study characteristics such as risk of bias. ${ }^{37}$ Another limitation of this review is that a similar systematic review was recently conducted. ${ }^{13}$ However, only seven studies were included in both reviews and the data included in this review tripled the evidence size compared with the previous (280532 more participants contributed date to our analysis than the 133861 participants previously). ${ }^{13}$ Yet one should bear in mind that the participants in this review primarily come from two large scale studies that both used data from Pregnancy Risk Assessment Monitoring System (PRAMS), ${ }^{21} 31$ while only one of these studies ${ }^{31}$ was included in the previous review. However, as there is no overlap in data-Silverman $e t a l^{31}$ used data from women participating in the PRAMS study between 2000 and 2003, whereas Wallenborn et $a l^{22}$ used data from women participating from 2004 to 2014, we considered them as separate studies - we believe it to be a strength of this review that both studies are included. In comparison to the other recent review, another strength of this review is that we conducted an appropriate quality assessment of all included studies and made use of a validated tool in the form of NOS, while Mezzavilla et $a l^{13}$ used 
Strengthening the Reporting of Observational Studies in Epidemiology (STROBE) to assess quality through bias susceptibility of included studies. However, STROBE is not an accepted quality assessment tool as this is a reporting guideline for observational studies ${ }^{3839}$ hence, the quality assessment conducted in this review is more meticulous. Yet, a limitation of NOS is that the quality assessors need to adapt the scale to specific research designs, which can lead to the possibility of low agreement between quality assessors. ${ }^{40}$ Nevertheless, as our quality assessment was conducted by two independent reviewers, we judged this issue to be minor. Further, the two versions of the NOS do not consider that cohort studies are superior to crosssectional studies in the evidence hierarchy, hence, this is a separate parameter to take into consideration when judging the overall quality of evidence according to NOS. Additionally, our review excluded studies with women who had a lifetime history of violence and childhood abuse, whereas the previous review included these populations of women. Hence, our exposure differs to some extent and a more heterogeneous exposure that consists of both childhood abuse and pregnancy-related IPV adds a further complicating element to the association.

\section{Interpretation of findings}

Overall, our study results support the findings of the recent review by Mezzavilla et $a l^{13}$ despite our review is mainly being based on different studies and have different exposures. In line with Mezzavilla et al we found that the most investigated outcome was exclusive breastfeeding, and that studies varied in quality. In contrast to Mezzavilla et al they also reported significant results from studies investigating women exposed to lifetime history of IPV. This may indicate that exposure to any time of violence may affect breastfeeding patterns. However, the reason why we choose to exclude lifetime IPV is that evidence points out that the association of only experiencing violence in pregnancy may be overestimated as there is evidence that victimisation as a young child increases the risk of further victimisation later in life, ${ }^{42}$ hence, it also increases risk of breastfeeding difficulties when becoming a mother. ${ }^{43}{ }^{44}$ Mediational models exploring childhood abuse and the negative association with breastfeeding have found it to stem from shame and the reaction to touch, in the postnatal period, which can lead to possible retraumatisation. ${ }^{10}$ With this in mind, it is interesting that only two studies in our review adjusted for childhood abuse in their statistical calculations with contradictory results. ${ }^{20}{ }^{35}$ Hence, as the majority of studies did not control for this factor, we cannot rule out that the exposure of IPV found in this study may be overestimated. Further, it is plausible that our exposure can be affected by recall bias. Women are primarily interviewed about exposure of IPV in relation to pregnancy in the postpartum period, which can potentially introduce recall bias as some women may not remember the extent of the violence or when they were exposed to violence. Moreover, women exposed to violence often under-report or refuse to participate in IPV studies in order to protect themselves or the perpetrator. ${ }^{45}$ If our effect estimates are affected by recall bias or under-reporting, it is plausible that true association is underestimated. Further, exclusive breastfeeding is often referred to as the most favourable type of feeding of infants. These recommendations may influence the women's reports on exclusive breastfeeding as is it can be strongly correlated to the feeling of being a 'good' mother. If women systematically erroneously report to exclusively breastfeed their babies to a higher extent than what to be the case, it is a type of reporting bias, which may also underestimate the true association between IPV and breastfeeding.

In relation to confounders it is worth noticing that our synthesis elucidated the comprehensive number of confounders that are adjusted for in the IPV - breastfeeding relationship. A key finding of this review is that most studies did not state their reasons for choice of confounders and there seems to be lack of consensus in the identification of potential confounders. Some covariates may be part of the causal pathway of the association between violence and breastfeeding, hence they are not true confounders. For instance, depression is one variable that can both be identified as a confounder, or an intermediate variable in the causal pathway. Sorbo et $a l^{35}$ concluded that depression could not explain early cessation of breastfeeding, while other studies ${ }^{4647}$ found that depression had a negative impact on breastfeeding duration in women suffering from depression. The mechanism between breastfeeding and depression is poorly understood, but research of failed lactation and perinatal depression theorise that it may be the manifestation of neuroendocrine perturbations in gonadal and lactogenic hormones. ${ }^{48}$ The lack of consensus in identification of potential confounders and their influence on the association between IPV and breastfeeding is also illustrated in two large scale studies by Wallenborn $e t a t^{22}$ and Silverman $e t a l .{ }^{31}$ Hence, Wallenborn $e t a l^{22}$ adjusted for marital status, education and insurance status, while Silverman $e t a l^{31}$ adjusted for race, age, marital status, education and smoking. Their data were from the same surveillance project (PRAMS), but interestingly, Silverman $e t a l^{31}$ did not find any significant association when controlling for confounders, opposite Wallenborn et $a l,{ }^{22}$ who found a significant association, but also found that stress and smoking affected breastfeeding when controlling for IPV, which provides evidence that stress and smoking are mediators and should not be treated as confounders.

Overall, the inconsistency of potential confounders proposes a need for defining core outcome measures related to IPV and breastfeeding practices. ${ }^{49}$ We suggest an individual patient data meta-analysis, ${ }^{50}$ by sharing raw data from existing studies and a powerful reanalysis adjusting for predefined confounders, can make evidence synthesis more robust in this area. 


\section{CONCLUSION}

This review shows that the association between IPV and breastfeeding is complex and that the effect of exposure to IPV on breastfeeding practices was difficult to properly assess based on data synthesis without the possibility of meta-analysis. The majority of studies in this review indicated that IPV exposure in pregnancy was associated with impaired breastfeeding, yet still some studies also found no association. There is no consensus of which confounders influence the relationship, hence, future research should aim to define core outcome measures and include longitudinal studies of high quality with predefined confounders.

\section{Twitter Ditte Søndergaard Linde @ditte_linde}

Acknowledgements Research librarians, Lasse Østengaard and Peter Everfelt, for assistance in literature search. Professor Stella Martin-de-Las-Heras for providing an advanced copy of her paper for inclusion in our review.

Contributors $A K N$ and $A B$ made the protocol and screened for eligible articles. AKN planned the data extraction, which was cross-checked and verified by FKM. AKN, FKM and DSL did quality assessment. Disagreement was solved through discussion. AKN designed the tables and wrote the first draft of the manuscript, which was reviewed by DSL, VR, KSK and AB. All authors approved the final manuscript.

Funding All authors were financed through their institutions: Department of Clinical Research, University of Southern Denmark (SDU) and Odense University Hospital (OUH). KSK is distinguished investigator at the University of Granada through a Beatriz Galindo (Senior Modality) Program grant of the Spanish Ministry of Science, Innovation and Universities.

Disclaimer The funders had no role in developing the article.

Competing interests DSL, AKN, AB and KSK had no conflict of interest. VR and FKM are coauthors of one study included in this review.

Patient consent for publication Not required.

Provenance and peer review Not commissioned; externally peer reviewed.

Data availability statement Data are available upon reasonable request.

Supplemental material This content has been supplied by the author(s). It has not been vetted by BMJ Publishing Group Limited (BMJ) and may not have been peer-reviewed. Any opinions or recommendations discussed are solely those of the author(s) and are not endorsed by BMJ. BMJ disclaims all liability and responsibility arising from any reliance placed on the content. Where the content includes any translated material, BMJ does not warrant the accuracy and reliability of the translations (including but not limited to local regulations, clinical guidelines, terminology, drug names and drug dosages), and is not responsible for any error and/or omissions arising from translation and adaptation or otherwise.

Open access This is an open access article distributed in accordance with the Creative Commons Attribution Non Commercial (CC BY-NC 4.0) license, which permits others to distribute, remix, adapt, build upon this work non-commercially, and license their derivative works on different terms, provided the original work is properly cited, appropriate credit is given, any changes made indicated, and the use is non-commercial. See: http://creativecommons.org/licenses/by-nc/4.0/.

\section{ORCID iDs}

Anne Katrine Normann http://orcid.org/0000-0003-1587-3616

Ditte Søndergaard Linde http://orcid.org/0000-0002-0851-6760

\section{REFERENCES}

1 Claudia Carcia-Moreno AG, Knerr W. Understanding and addressing violence against women WHO, 2012.

2 WHO. Violence against women, 2017. Available: https://www. who.int/en/news-room/fact-sheets/detail/violence-against-women [Accessed 10 Apr 2019].

3 World Health Organization/London School of Hygiene and Tropical Medicine. Preventing intimate partner and sexual violence against women: taking action and generating evidence. Geneva, 2010.
4 WHO. Intimate partner violence during pregnancy. Department of Reproductive Health and Research, 2011.

5 Jasinski JL. Pregnancy and domestic violence: a review of the literature. Trauma Violence Abuse 2004:5:47-64.

6 Sigalla GN, Mushi D, Meyrowitsch DW, et al. Intimate partner violence during pregnancy and its association with preterm birth and low birth weight in Tanzania: a prospective cohort study. PLoS One 2017;12:e0172540

7 WHO. Improving maternal, newborn, infant and young child health and nutrition, 2013.

8 Chowdhury AN, Ramakrishna J, Chakraborty AK, et al. Cultural context and impact of alcohol use in the Sundarban delta, West Bengal, India. Soc Sci Med 2006;63:722-31.

9 Hasselmann MH, Werneck GL, Silva CVCda. Symptoms of postpartum depression and early interruption of exclusive breastfeeding in the first two months of life. Cad Saude Publica 2008;24:s341-52.

10 Wood K, Van Esterik P. Infant feeding experiences of women who were sexually abused in childhood. Can Fam Physician 2010;56:e136-41.

11 Coles J. Qualitative study of breastfeeding after childhood sexual assault. J Hum Lact 2009;25:317-24.

12 Misch ES, Yount KM. Intimate partner violence and breastfeeding in Africa. Matern Child Health J 2014;18:688-97.

13 Mezzavilla RdeS, Ferreira MdeF, Curioni CC, et al. Intimate partner violence and breastfeeding practices: a systematic review of observational studies. J Pediatr 2018;94:226-37.

14 Moher D, Liberati A, Tetzlaff J, et al. Preferred reporting items for systematic reviews and meta-analyses: the PRISMA statement. PLoS Med 2009;6:e1000097.

15 Stroup DF, Berlin JA, Morton SC, et al. Meta-analysis of observational studies in epidemiology: a proposal for reporting. meta-analysis of observational studies in epidemiology (MOOSE) group. JAMA 2000;283:2008-12.

16 Schardt C, Adams MB, Owens T, et al. Utilization of the PICO framework to improve searching PubMed for clinical questions. BMC Med Inform Decis Mak 2007;7:16.

17 Covidence systematic review software, veritas health innovation, Melbourne, Australia. Available: www.covidence.org [Accessed 25 Feb 2019].

18 Coomarasamy A, Khan KS. What is the evidence that postgraduate teaching in evidence based medicine changes anything? A systematic review. BMJ 2004;329:1017.

19 ter Riet G, Kleijnen J, Knipschild P. Acupuncture and chronic pain: a criteria-based meta-analysis. J Clin Epidemiol 1990;43:1191-9.

20 Miller-Graff LE, Ahmed AH, Paulson JL. Intimate partner violence and breastfeeding outcomes in a sample of low-income women. J Hum Lact 2018;34:494-502.

21 Tiwari S, Gray R, Jenkinson C, et al. Association between spousal emotional abuse and reproductive outcomes of women in India: findings from cross-sectional analysis of the 2005-2006 national family health survey. Soc Psychiatry Psychiatr Epidemiol 2018;53:509-19.

22 Wallenborn JT, Cha S, Masho SW. Association between intimate partner violence and breastfeeding duration: results from the 20042014 pregnancy risk assessment monitoring system. J Hum Lact 2018;34:233-41.

23 Boyce SC, McDougal L, Silverman JG, et al. Associations of intimate partner violence with postnatal health practices in Bihar, India. BMC Pregnancy Childbirth 2017;17:398.

24 Finnbogadóttir $\mathrm{H}$, Thies-Lagergren L. Breastfeeding in the context of domestic violence-a cross-sectional study. J Adv Nurs 2017;73:3209-19.

25 Holland ML, Thevenent-Morrison K, Mittal M, et al. Breastfeeding and exposure to past, current, and neighborhood violence. Matern Child Health J 2018;22:82-91.

26 Islam MJ, Baird K, Mazerolle P, et al. Exploring the influence of psychosocial factors on exclusive breastfeeding in Bangladesh. Arch Womens Ment Health 2017;20:173-88.

27 James JP, Taft A, Amir LH, et al. Does intimate partner violence impact on women's initiation and duration of breastfeeding? Breastfeed Rev 2014;22:11-19.

28 Moraes CL, de Oliveira ASD, Reichenheim ME, et al. Severe physical violence between intimate partners during pregnancy: a risk factor for early cessation of exclusive breast-feeding. Public Health Nutr 2011;14:2148-55.

29 Shroff MR, Griffiths PL, Suchindran C, et al. Does maternal autonomy influence feeding practices and infant growth in rural India? Soc Sci Med 2011;73:447-55.

30 Lau Y, Chan KS. Influence of intimate partner violence during pregnancy and early postpartum depressive symptoms on 
breastfeeding among Chinese women in Hong Kong. J Midwifery Womens Health 2007:52:e15-20.

31 Silverman JG, Decker MR, Reed E, et al. Intimate partner violence around the time of pregnancy: association with breastfeeding behavior. J Womens Health 2006;15:934-40.

32 Kjerulff Madsen F, Holm-Larsen CE, Wu C, et al. Intimate partner violence and subsequent premature termination of exclusive breastfeeding: a cohort study. PLoS One. In Press 2019;14:e0217479.

33 Martin-de-Las-Heras S, Velasco C, Luna-Del-Castillo JD, et al. Breastfeeding avoidance following psychological intimate partner violence during pregnancy: a cohort study and multivariate analysis. BJOG 2018.

34 Hasselmann MH, Lindsay AC, Surkan PJ, et al. Intimate partner violence and early interruption of exclusive breastfeeding in the first three months of life. Cad Saude Publica 2016;32:e00017816.

35 Sørbø MF, Lukasse M, Brantsæter A-L, et al. Past and recent abuse is associated with early cessation of breast feeding: results from a large prospective cohort in Norway. BMJ Open 2015;5:e009240.

36 Khan KS, Ball E, Fox CE, et al. Systematic reviews to evaluate causation: an overview of methods and application. Evid Based Med 2012;17:137-41.

37 Schriger DL, Altman DG, Vetter JA, et al. Forest plots in reports of systematic reviews: a cross-sectional study reviewing current practice. Int J Epidemiol 2010;39:421-9.

38 da Costa BR, Cevallos M, Altman DG, et al. Uses and misuses of the STROBE statement: bibliographic study. BMJ Open 2011;1:e000048.

39 von Elm E, Altman DG, Egger M, et al. The strengthening the reporting of observational studies in epidemiology (STROBE) statement: guidelines for reporting observational studies. Lancet 2007:370:1453-7.
40 Stang A. Critical evaluation of the Newcastle-Ottawa scale for the assessment of the quality of nonrandomized studies in metaanalyses. Eur J Epidemiol 2010;25:603-5.

41 Luchini C, Stubbs B, Solmi M, et al. Assessing the quality of studies in meta-analyses: advantages and limitations of the Newcastle Ottawa scale. World J Metaanal 2017;5:80.

42 Widom CS, Czaja SJ, Dutton MA. Childhood victimization and lifetime revictimization. Child Abuse Negl 2008;32:785-96.

43 Elfgen C, Hagenbuch N, Görres G, et al. Breastfeeding in women having experienced childhood sexual abuse. J Hum Lact 2017;33:119-27.

44 Eagen-Torkko M, Low LK, Zielinski R, et al. Prevalence and predictors of breastfeeding after childhood abuse. J Obstet Gynecol Neonatal Nurs 2017;46:465-79.

45 Gracia E. Unreported cases of domestic violence against women: towards an epidemiology of social silence, tolerance, and inhibition. $J$ Epidemiol Community Health 2004;58:536-7.

46 Henderson JJ, Evans SF, Straton JAY, et al. Impact of postnatal depression on breastfeeding duration. Birth 2003;30:175-80.

47 Hatton DC, Harrison-Hohner J, Coste S, et al. Symptoms of postpartum depression and breastfeeding. J Hum Lact 2005;21:444-9.

48 Stuebe AM, Grewen K, Pedersen CA, et al. Failed lactation and perinatal depression: common problems with shared neuroendocrine mechanisms? J Womens Health 2012;21:264-72.

49 Williamson PR, Altman DG, Bagley H. The comet Handbook: version 1.0 the comet Handbook: version 1.0, 2017.

50 Rogozińska E, Marlin N, Thangaratinam S, et al. Meta-Analysis using individual participant data from randomised trials: opportunities and limitations created by access to RAW data. Evid Based Med 2017;22:157-62. 